\title{
Efeito da Linhagem e do Nível de Lisina da Dieta sobre a Qualidade da Carne do Peito de Frangos de Corte
}

\author{
Ana Cláudia Pavan¹, Ariel Antônio Mendes ${ }^{2}$, Edson Gonçalves de Oliveira ${ }^{3}$, Juliana Célia \\ Denadai ${ }^{1}$, Rodrigo Garófallo Garcia ${ }^{4}$, Tania Sayuri Takita ${ }^{5}$
}

\begin{abstract}
RESUMO - O experimento foi conduzido com o objetivo de avaliar o efeito da linhagem e do nível de lisina da dieta sobre o rendimento e a qualidade da carne de peito de frangos de corte. Foram utilizados 2250 pintos não sexados, de três linhagens comerciais, identificadas como A, B e C, distribuídos em delineamento inteiramente casualizado em esquema fatorial 3x3 (linhagens e níveis de lisina), com cinco repetições de 50 aves. As aves receberam ração à vontade, à base de milho e farelo de soja, suplementadas com 1,18; 1,24 e 1,30\% de lisina nas rações pré-iniciais ( 1 a 7 dias); 1,10; 1,16 e 1,22\% nas rações iniciais ( 7 a 21 dias); 1,0; 1,06 e 1,12\% nas rações de crescimento (21 a 35 dias) e 0,$85 ; 0,91$ e 0,97\% nas rações de terminação (35 a 42 dias). Foram avaliadas as características de rendimento de peito e carne de peito e as medidas de espessura, largura, comprimento, $\mathrm{pH}$, perda de peso por cozimento e força de cisalhamento da carne de peito. Para o rendimento de peito não foram observadas diferenças significativas, enquanto para rendimento de carne de peito houve efeito significativo da linhagem, em que a linhagem A apresentou o maior rendimento e a linhagem B, o menor. Para as medidas físicas da carne do peito, foram encontradas diferenças significativas entre as linhagens para comprimento de peito, com a linhagem $\mathrm{C}$ apresentando o maior resultado. $\mathrm{O}$ pH da carne diferiu significativamente para linhagens e níveis de lisina. As linhagens tiveram efeito sobre o rendimento, comprimento e $\mathrm{pH}$ da carne de peito, em que a linhagem A apresentou maiores valores para rendimento e $\mathrm{pH}$ e a linhagem C para comprimento.
\end{abstract}

Palavras-chave: frangos de corte, lisina, peito, $\mathrm{pH}$, rendimento

\section{Effect of Strain and Dietary Lysine Level on Breast Meat Quality of Broiler Chickens}

ABSTRACT - The objective of this experiment was to evaluate the effect of broiler strain and dietary lysine level on breast meat yield and quality. Two thousand two hundred and fifty day old straightrun chicks from commercial strains named A, B and C were reared in a factorial $3 \times 3$ arrangement (strain and dietary lysine level) with five replicates of 50 birds per pen. The broiler chikens were feed ad libitun with a corn and soybean diet withing the following lysine levels: $1.18,1.24$ and 1.30\% in pre-starter diets (1-7 days); $1.10,1.16$ and $1.22 \%$ in starter diets (7-21 days); $1.00,1.06$ and 1.12\% in grower diets (21-35 days) and .85, .91 and .97\% in finishing diets (3542 days). Total breast yield, breast meat yield, $\mathrm{pH}$, breast measures (high, width and lenght), weight cooking loss, and breast meat shear force were evaluated. There were no effect of strain and diet on breast yield. Related to breast meat yield, strain A presented higher values than strain B. Strain C presented the highest breast lenght. There were effects of lysine level only for $\mathrm{pH}$. $\mathrm{pH}$ was affected by strain and dietary lisine level. It was concluded that lysine level affected only pH value. Strain affected breast meat yield, lenght and pH, where strain A showed the highest values for yield and $\mathrm{pH}$ and strain $\mathrm{C}$ for breast lenght.

Key Words: breast, broiler, lysine, $\mathrm{pH}$, yield

\section{Introdução}

Nos últimos anos, ocorreram modificações na comercialização da carne de aves, aumentando a exportação e o consumo interno de partes desossadas. Com isso, o rendimento de cortes nobres, como peito e pernas, tornou-se importante para a indús- tria. O peito é utilizado na elaboração de vários produtos pós-processados e, por isso, é natural que as integrações estejam preocupadas com sua qualidade e rendimento.

Mendes (1990) e Mendes et al. (1996) afirmaram que a quantidade de carne produzida pode ser aumentada na dependência dos níveis de nutrientes

\footnotetext{
${ }^{1}$ Aluna de Pós-Graduação em Zootecnia (Mestrado) da Faculdade de Medicina Veterinária e Zootecnia - UNESP, Campus de Botucatu. E.mail: ana.pavan@fca.unesp.br; juliana.denadai@bol.com.br

2 Docente do Departamento de Produção e Exploração Animal da Faculdade de Medicina Veterinária e Zootecnia - UNESP, Campus de Botucatu. E.mail: arielmendes@fca.unesp.br

3 Professor do Departamento de Zootecnia da Universidade Federal do Paraná. E.mail: ego@ufpr.br

${ }^{4}$ Aluno de Pós-Graduação em Zootecnia (Doutorado) da Faculdade de Medicina Veterinária e Zootecnia - UNESP, Campus de Botucatu. E.mail: garofallo@fca.unesp.br

${ }^{5}$ Aluna de Pós-Graduação em Genética (Doutorado) do Instituto de Biociências - UNESP, Campus de Botucatu.
} 
da dieta, principalmente lisina e metionina. Lubritz (1997) e Robinson et al. (1996) verificaram que o comprimento, espessura, largura e peso de filés de peito podem ser afetados pela linhagem, sexo e idade das aves. Baker (1995) observou que as diferenças entre linhagens quanto às exigências de lisina são devidas às diferenças na composição corporal, ou seja, se determinada linhagem apresenta exigência maior de proteína e menor de gordura, poderia apresentar exigência maior de lisina.

A perda de peso por cozimento nos músculos do peito de frangos é uma característica que, de acordo com alguns autores, pode ser significativamente influenciada pelas temperaturas elevadas durante o período de criação e no período pré-abate. Bressan (1998) observou que peitos de aves mantidas em ambientes com temperatura de $30^{\circ} \mathrm{C}$ apresentaram maior perda de peso por cozimento, com média de $28,7 \%$, quando comparadas com os peitos de aves que foram submetidas a ambientes de conforto térmico $\left(17,1^{\circ} \mathrm{C}\right)$, com média de $27,2 \%$. Resultados semelhantes foram encontrados por Kim et al. (1988), que observaram que os valores de $\mathrm{pH}$ do peito de frangos submetidos ao estresse calórico, eram mais baixos aos 30 minutos pós morte e que a perda de peso por cozimento do peito foi significativamente maior $(\mathrm{p}<0,05)$ nas aves estressadas pelo calor.

Com base nestes aspectos, o objetivo deste trabalho foi avaliar o efeito da linhagem e dos níveis de lisina da dieta sobre o rendimento do peito e a qualidade do peito de frangos de corte.

\section{Material e Métodos}

Foram utilizados 2250 pintos de um dia, não sexados, das três linhagens comerciais de frangos de corte: Ross 308, Ross 508 e Cobb 500, identificadas como A, B e C, alimentados com dietas contendo três níveis de lisina. Os ovos eram provenientes de matrizes com aproximadamente 40 semanas de idade, criadas na mesma granja e incubados em uma mesma máquina incubadora. $\mathrm{O}$ delineamento experimental adotado foi o inteiramente casualizado em esquema fatorial $3 \times 3$ (três linhagens e três níveis de lisina), com cinco repetições de 50 aves cada. As aves receberam ração à vontade à base de milho e farelo

Tabela 1 - Composição percentual e níveis nutricionais das dietas basais Table 1 - Percentual composition and nutritional levels of the basal diets

\begin{tabular}{|c|c|c|c|c|}
\hline $\begin{array}{l}\text { Ingrediente } \\
\text { Ingredient }\end{array}$ & $\begin{array}{l}\text { Pré-inicial } \\
\text { Pre-starter }\end{array}$ & $\begin{array}{l}\text { Inicial } \\
\text { Starter }\end{array}$ & $\begin{array}{c}\text { Crescimento } \\
\text { Grower }\end{array}$ & $\begin{array}{c}\text { Final } \\
\text { Finishing }\end{array}$ \\
\hline Milho moído (Ground corn) & 55,57 & 59,16 & 63,14 & 69,50 \\
\hline Farelo de soja (Soybean meal) & 37,09 & 34,00 & 30,00 & 24,01 \\
\hline Fosfato bicálcico (Dicalcium phosphate) & 1,75 & 1,70 & 1,65 & 1,45 \\
\hline Calcário calcítico (Limestone) & 2,10 & 1,15 & 1,15 & 1,05 \\
\hline Caulin (Caulin) & 0,30 & 0,30 & 0,30 & 0,30 \\
\hline $\operatorname{Sal}($ Salt $)$ & 0,40 & 0,43 & 0,41 & 0,39 \\
\hline Óleo vegetal (Vegetable oil) & 1,60 & 2,10 & 2,20 & 2,20 \\
\hline Suplemento vitamínico (Vitamin premix) & 0,50 & 0,50 & 0,50 & 0,50 \\
\hline Suplemento mineral (Mineral Premix) & 0,50 & 0,50 & 0,50 & 0,50 \\
\hline DL-metionina (DL-Methionine) & 0,19 & 0,17 & 0,15 & 0,10 \\
\hline \multicolumn{5}{|l|}{$\begin{array}{l}\text { Níveis nutricionais calculados } \\
\text { Calculated nutritional levels }\end{array}$} \\
\hline Energia metabolizável (kcal/kg) ME (kcal/kg) & 2950 & 3050 & 3100 & 3180 \\
\hline Proteína bruta (\%) (Crude protein, \%) & 22,00 & 20,85 & 19,38 & 17,17 \\
\hline Lisina (Lysine), $(\%)$ & 1,18 & 1,10 & 1,00 & 0,85 \\
\hline Metionina + Cistina $(\%)$ (Methionine + Cystine) & 0,90 & 0,85 & 0,79 & 0,68 \\
\hline Cálcio (Calcium) \% & 1,10 & 1,00 & 0,95 & 0,85 \\
\hline Sódio(Sodium) (\%) & 0,25 & 0,20 & 0,19 & 0,18 \\
\hline Fósforo Total (Total phosphorus) \% & 0,68 & 0,67 & 0,64 & 0,59 \\
\hline
\end{tabular}

1 Composição por kg (composition per kg) das rações pré-inicial (pre-starter), inicial (starter), crescimento (grower) e final (finishing), respectivamente: vit. A - 8.000, 8.000, 6.400 e 5.200 UI; Vitamina $D^{3}-3.510$, 3.510, 2.810 e 2.250 UI; Vitamina E- 15, 15, 12 e 9,6 mg; Vitamina K- 3, 3, 2,4 e 1,92 mg; Tiamina (Tiamine)- 2,35, 2,33, 2,10 e 1,89mg; Riboflavina (Riboflavin)- 6,54, 6,47, 5,38 e 4,44 mg; Piridoxina (Piridoxin)- 5,91, 5,90, 5,45 e 5,08mg; Vitamina B 12- 12, 12, 9,6 e 7,68 mcg; Niacina (Niacin)- 41,15, 40,53, 33,75 e 27,79 mg; Ácido Pantotenico (Pantothenic acid)- 15,26, 15,02, 12,71 e 10,66 mg; Ácido fólico (Folic acid) - 2,11, 2,06, 1,75 e 1,46 mg; Biotina (Biotin)- 0,20, 0,20, 0,16 e 0,13 mg; Cloreto de colina (Choline chloride) - 1,47, 1,42, 1,29 e 1,08 g; Suplemento mineral (Mineral premix): Ferro (Iron)- 121,25, 119,44, 115,11 e 105,55 mg; Cobre (Copper) - 106, 106, 106 e 6 mg; Manganês (Manganese) - 72,25, 72,02, 71,47 e 70,25 mg; Zinco (Zinc)60, 60, 60 e 60 mg; lodo (Iodine) - 1,2, 1,2, 1,2 e 1,2 mg; Selênio (Selenium) - 0,4, 0,4, 0,4 e 0,13 mg. 
de soja suplementadas com $1,18,1,24$ e $1,30 \%$ de lisina nas rações pré-iniciais; $1,10,1,16$ e 1,22\% nas rações iniciais; $1,0,1,06$ e 1,12\% nas rações de crescimento e 0,$85 ; 0,91$ e $0,97 \%$ nas rações finais. As rações basais são apresentadas na Tabela 1, sendo que os níveis de lisina foram obtidos com a substituição de substância inerte (Caulin) por L-Lisina (99\%). Os níveis recomendados pelo NRC (1994) de 1,$18 ; 1,10 ; 1,00$ e $0,85 \%$ foram considerados baixos, para rações pré-inicial, inicial, crescimento e final, respectivamente. Os demais níveis foram considerados como intermediários e altos.

Foram avaliadas as características de rendimento de peito e carne de peito, $\mathrm{pH}$, comprimento, largura, espessura, perda de peso por cozimento e força de cisalhamento da carne de peito. Aos 42 dias de idade foi retirada uma amostra de 6 aves por repetição, as quais foram abatidas. $\mathrm{O}$ rendimento de peito e carne de peito foi realizado de acordo com Mendes (1990). $\mathrm{O}$ pH da carne foi obtido 24 horas post mortem através de eletrodo de penetração. As amostras de peito foram congeladas a $-20^{\circ} \mathrm{C}$ e posteriormente foi retirado o músculo pectoralis major, no qual foi realizado as medidas de comprimento e largura (lados esquerdo e direito) com o auxílio de uma régua, e a espessura (lados esquerdo e direito) com o auxílio de um paquímetro.

Para determinação da perda de peso por cozimento e da força de cisalhamento foi utilizado o músculo peitoral esquerdo da ave, o qual foi embalado em papel laminado e mantido numa chapa elétrica de modelo comercial, com aquecimento nas duas faces, por aproximadamente 8 minutos a $85^{\circ} \mathrm{C}$. Depois de uma hora, a amostra de peito foi pesada, obtendo-se assim o peso 1 hora após o cozimento. As amostras foram embaladas em papel absorvente e em sacos plásticos. Após 24 horas, as amostras foram desembaladas e novamente pesadas, obtendo-se o peso 24 horas após o cozimento. A diferença de peso entre peso do peito in natura e o peso cozido com 1 e 24 horas após o cozimento correspondem à perda de peso por cozimento nos dois períodos (Honikel, 1987). Estas mesmas amostras foram utilizadas para determinação da força de cisalhamento, para a qual foram retiradas 6 a 8 amostras na forma de paralelepípedos com medidas $2 \times 2 \times 1,13 \mathrm{~cm}$, as quais foram colocadas com as fibras orientadas no sentido perpendicular às lâminas do aparelho Warner-Bratzler, acoplado ao aparelho Instron M 2318, conforme técnica descrita por Froming et al. (1978).

\section{Resultados e Discussão}

Os resultados observados estão apresentados nas Tabelas 2 e 3.

Para o rendimento de peito, não foram observadas diferenças significativas ( $p>0,05)$ entre os níveis de lisina e linhagens. Resultados semelhantes foram encontrados por Acar et al. (1991), que, ao avaliarem o rendimento de carcaça de frangos de corte machos de duas linhagens comerciais, recebendo rações com nível de lisina acima e abaixo do recomendado pelo NRC (1994), não encontraram diferenças significativas para rendimento de peito. O rendimento de carne de peito apresentou efeito significativo $(p<0,05)$ para linhagens, onde as linhagens A e C

Tabela 2 - Rendimento de peito e carne de peito de frangos de corte de três linhagens comerciais alimentados com rações contendo diferentes níveis de lisina

Table 2 - Total breast and breast meat yield of broiler chickens of three commercial strains fed diets with different lysine levels

\begin{tabular}{lccc}
\hline & $\begin{array}{c}\text { Peito } \\
(\%) \\
\text { Breast }\end{array}$ & $\begin{array}{c}\text { Carne de } \\
\text { peito }^{2}(\%) \\
\text { Breastmeat }\end{array}$ \\
\hline Linhagem & $\mathrm{A}$ & 24,13 & $18,89^{\mathrm{a}}$ \\
Strain & $\mathrm{B}$ & 23,38 & $18,08^{\mathrm{b}}$ \\
& $\mathrm{C}$ & 23,56 & $18,25^{\mathrm{ab}}$ \\
Níveis & Baixo & 23,51 & 18,18 \\
de lisina & Low & & 18,63 \\
Lysine levels & Intermediate & 23,71 & 18,39 \\
& Alto & 23,82 & \\
\hline 1 & High & & \\
\hline
\end{tabular}

${ }_{1}^{1}$ Peito: rendimento de peito em relação à carcaça inteira.

${ }^{2}$ Carne de peito: rendimento de carne de peito em relação à carcaça inteira.

Médias seguidas de letras diferentes na mesma coluna diferem $(P<0,05)$ pelo teste Tukey.

1 Breast: breast yield in relation to the total carcass weight.

${ }^{2}$ Breast meat: breast meat yield in relation to the total carcass weight. Means within a columm, followed by different letter, are different $(p<.05)$ by Tukey test. 
apresentaram os maiores valores, sendo que $\mathrm{C}$ não diferiu de A. Para $\mathrm{pH}$ da carne observou-se efeito significativo $(p<0,05)$ da linhagem e níveis de lisina, sendo que as linhagens $\mathrm{A}$ e B apresentaram os maiores valores e a $\mathrm{B}$ não diferiu da $\mathrm{C}$. Com relação aos níveis de lisina, os maiores valores de $\mathrm{pH}$ foram observados com os níveis intermediários e altos. Valores semelhantes foram encontrados por Jones \& Grey (1989) e Sams \& Mills (1993).

Robinson et al. (1996) avaliaram a influência da linhagem, sexo e idade no desenvolvimento do músculo do peito de frangos de corte e encontraram que não houve diferenças significativas entre linhagens para a área e comprimento do músculo pectoralis major. Já para o músculo pectoralis minor, foram encontradas somente diferenças de peso.

Os autores concluíram que o incremento da massa muscular do peito é altamente correlacionado à espessura do pectoralis major, porém com menor aumento da área.

Os mesmos autores também avaliaram o peso, a área e a espessura da carne de peito de quatro linhagens de frangos de corte e encontraram diferença significativa para a espessura, mas não para o comprimento do pectoralis major entre linhagens.

Para as medidas físicas do peito observou-se efeito significativo $(\mathrm{p}<0,05)$ de linhagens apenas para o comprimento do peito, sendo que a linhagem $\mathrm{C}$ apresentou os maiores valores para comprimento do lado direito e esquerdo e as linhagens A e B apresentaram os menores valores, os quais não diferiram entre si.

A maciez da carne foi medida pela força de cisalhamento. Conforme Froming et al. (1978), a maciez da carne de peito de frangos de corte está associada à capacidade de retenção de água apresentada pelo músculo, a qual é dependente de vários fatores como o estresse térmico sofrido pela ave durante o período de criação e os fatores pré-abate.

A textura (maciez) da carne é o critério de qualidade mais importante e pode ser avaliada por métodos subjetivos e métodos objetivos, como a medição do $\mathrm{pH}$ da carne, perda de peso por cozimento e força de cisalhamento (Bressan, 1998).

Na literatura há discordância em relação a valores limites de força de cisalhamento para considerar a carne de peito de frango como macia. Lyon et al. (1995) utilizaram como referência o valor de 7,5 kgf/g, enquanto Simpson et al. (1992), de 8,0 kgf/g como limite, acima do qual a carne seria considerada dura.

Nas condições experimentais utilizadas neste trabalho, os valores de força de cisalhamento entre 1,91 e 2,23 encontram-se perfeitamente na faixa de variação que considera a carne macia.

Em relação ao valor de $\mathrm{pH}$ final em peito de frangos os autores divergem. Mellor et al. (1958) observaram valores médios mais elevados, entre 5,9 e 6,2. Já Jones \& Grey (1989) observaram valores

Tabela 3 - Qualidade da carne de peito de frangos de corte de três linhagens alimentados com rações contendo diferentes níveis de lisina

Table 3 - Breast meat qualiy parameters of broiler chickens of three stains fed diets with different lysine levels

\begin{tabular}{|c|c|c|c|c|c|c|}
\hline \multirow[b]{2}{*}{$\begin{array}{l}\text { Variáveis } \\
\text { Variables }\end{array}$} & \multicolumn{3}{|c|}{$\begin{array}{l}\text { Linhagens } \\
\text { Strains }\end{array}$} & \multicolumn{3}{|c|}{$\begin{array}{l}\text { Níveis de lisina } \\
\text { Lysine levels }\end{array}$} \\
\hline & A & B & $\mathrm{C}$ & $\begin{array}{l}\text { Baixo } \\
\text { Low }\end{array}$ & $\begin{array}{l}\text { Intermediário } \\
\text { Intermediate }\end{array}$ & $\begin{array}{l}\text { Alto } \\
\text { High }\end{array}$ \\
\hline $\begin{array}{l}\text { Altura (cm) } \\
\text { High }\end{array}$ & 2,40 & 2,32 & 2,34 & 2,31 & 2,35 & 2,33 \\
\hline $\begin{array}{l}\text { Largura }(\mathrm{cm}) \\
\text { Width }\end{array}$ & 7,61 & 7,57 & 7,61 & 7,57 & 7,64 & 7,57 \\
\hline $\begin{array}{l}\text { Comprimento }(\mathrm{cm}) \\
\text { Length }\end{array}$ & $17,33^{\mathrm{b}}$ & $17,36^{\mathrm{b}}$ & $17,84^{\mathrm{a}}$ & 17,37 & 17,64 & 17,53 \\
\hline $\mathrm{pH}$ & $6,01^{\mathrm{a}}$ & $5,99^{\mathrm{ab}}$ & $5,94^{\mathrm{b}}$ & $5,94^{\mathrm{b}}$ & $6,01^{\mathrm{a}}$ & $5,98^{\mathrm{ab}}$ \\
\hline $\begin{array}{l}\text { Força de cisalhamento }(\mathrm{kgf} / \mathrm{g}) \\
\text { Shear force }\end{array}$ & 1,95 & 2,09 & 2,11 & 2,23 & 1,91 & 2,13 \\
\hline $\begin{array}{l}\text { Perda de peso por cozimento }(\mathrm{g}) \\
\text { Weight loss }\end{array}$ & 18,44 & 18,16 & 19,44 & 17,75 & 19,68 & 19,54 \\
\hline $\begin{array}{l}\text { Perda de peso por cozimento (g) } 24 \text { horas } \\
\text { Weight loss }\end{array}$ & 22,23 & 21,07 & 22,63 & 21,40 & 22,13 & 22,40 \\
\hline
\end{tabular}

Médias seguidas de letras diferentes na linha e dentro de cada fator diferem $(P<0.05)$ entre si pelo teste Tukey.

Means followed by the same letters in the row and inside the parameter are different $(p<.05)$ by Tukey test. 
médios de pH entre 5,6 e 5,8 e Sams \& Mills (1993) reportaram resultados entre 5,78 e 5,86. Os valores encontrados neste experimento estão dentro da faixa de variação de $\mathrm{pH}$ descritos na literatura.

Para perda de peso por cozimento, Dunn et al. (1993) encontraram variações médias de 18,04 a 20,55g, enquanto Bilgili et al. (1989), valores de 25,1 a $29 \mathrm{~g}$ (ou superiores). No presente trabalho foram encontrados valores entre 18,4 e 19,5g, concordando com os obtidos pelos primeiros autores.

\section{Conclusões}

Não foram encontradas diferenças significativas entre as linhagens e os níveis de lisina para rendimento de peito. Quanto ao rendimento da carne de peito, houve diferença entre as linhagens, com a linhagem A apresentando o maior valor e não diferindo estatisticamente da linhagem C. Não houve efeito dos níveis de lisina para rendimento de carne de peito.

Para os parâmetros de qualidade de carne de peito, somente foram encontradas diferenças significativas quanto ao comprimento do peito para as linhagens e ao $\mathrm{pH}$ para linhagens e níveis de lisina. A linhagem $\mathrm{C}$ apresentou o maior valor de comprimento de peito. A linhagem A apresentou o maior valor de $\mathrm{pH}$, que não diferiu significativamente da linhagem B, enquanto, para níveis de lisina, o maior valor de $\mathrm{pH}$ foi obtido com o nível intermediário, o qual não diferiu do nível alto.

Com base nos dados obtidos neste trabalho, pode-se inferir que a linhagem $\mathrm{C}$ apresentou os melhores resultados frente aos parâmetros estudados e o melhor nível de lisina encontrado foi o intermediário.

\section{Literatura Citada}

ACAR, N.; MORAN, E.T.; BILGILI, S.F. Live performance and carcass yield of male broilers from two commercial strain crosses receiving rations containing lysine below and above the stablished requirement between 6 and 8 weeks of age. Poultry Science, v.70, n.11, p.2315-2331, 1991.

BAKER, H.D. Ideal protein for broiler chicks. In: MULTISTATE POULTRY FEEDING AND NUTRITION CONFERENCE, 12., 1995, Indianópolis. Proceedings... Indianápolis: PSA, 1995. p.1-13.

BILGILI, S.F.; EGBERT, W.R.; HOFFMAN, D.L. Effect of posmortem ageing temperature on sarcomere lengh and tenderness of broiler pectoralis major. Poultry Science, v.68, n.11, p.1588-1591, 1989.
BRESSAN, C. Efeito dos fatores pré-abate sobre a qualidade do peito de frango. Campinas: Faculdade de Engenharia de Alimentos, 1998. 179p. Tese (Doutorado em Zootecnia) Faculdade de Engenharia de Alimentos/ Unicamp, 1998.

DUNN, A.A.; KILPATRICS, D.J.; GAULT, N.F.S.E. Effect of post mortem temperature on chicken pectoralis major : muscle shortening and cooked meat tenderness. British Poultry Science, v.34, n.4, p.689-697, 1993.

FROMING, G.W.; BABJI, A.S.; MATHER, F.B. The effect of preslaughter temperatures, stress, struggle and anesthetiztion on color and textural characterisitcs of turkey muscle. Poultry Science, v.57, n.3, p.630-633, 1978.

HONIKEL, K.O. The water binding of meat. Fleischwirtschaft, v.67, p.1098-1102, 1987.

JONES, J.M.; GREY, T.C. Influence of processing on product quality and yield. Processing of Poultry, p.127-130, 1989.

KIM, J.W.; FLETCHER, D.L.; CAMPION, D.R. Effect of eletrical stunning and hot deboning on broiler breast meat characteristics. Poultry Science, v.67, n.4, p.674-676, 1988.

LYON, C.E.; HAMM, D.E.; THOMSON, J.E. pH and tenderness of broiler breast meat deboned various times after chilling. Poultry Science, v.64, n.2, p.307-310, 1985.

LUBRITZ, S.L. A statistical model for white meat yield in broiler. Journal of Applied Poultry Research, v.6, n.3, p.253-259, 1997.

MELLOR, D.B.; SRINGER, P.A.; MOUNTNEY, G.J. The influence of glicogen on tenderness of broiler meat. Poultry Science, v.37, n.3, p.1028-1029, 1958.

MENDES, A.A. Efeito de fatores genéticos, nutricionais e de ambiente sobre o rendimento de carcaça de frangos de corte. Botucatu: Universidade Estadual Paulista, 1990. 103p. Tese (Livre Docência) - Universidade Estadual Paulista, 1990.

MENDES, A.A.; WATKINS, S.E.; ENGLAND, J.A. et al. Influence of arginine : lysine ratios on performance of broilers during heat stress and cold stress. Poultry science, v.76, n.3, p.472-481, 1997.

NATIONAL RESEARCH COUNCIL - NRC. Nutrient requeriments of poultry. 9.rev.ed. Washington: National Academy Press, 1994. 156p.

ROBINSON, F.E.; ROBINON, N.A.; TURNER, B.V. et al. Breast muscle development in broilers as affected by strain, sex and age at processing. In: POULTRY SCIENCE ANNUAL MEETING, 85., 1996, Louiseville. Proceedings... Louiseville: PSA, 1996. p.56.

SAMS, A.R.; MILLS, K.A. The effect of feed withdrawal duration on the responsiveness of broiler pectoralis to rigor mortis acceleration. Poultry Science, v.72, n.9, p.1789-1796, 1993.

SIMPSON, M.D.; GOODWIN T.L. Comparison between shear values and test panel scores for predicting tenderness of broilers. Poultry Science, v.53, n.6, p.2042-2046, 1974.
Recebido em: 06/05/02 Aceito em: 03/04/03 